\title{
Characterization of Single Barrier Microrefrigerators at Cryogenic Temperatures
}

\author{
X. WANG ${ }^{1,3}$ Y. EZZAHRI ${ }^{1}$ Z. BIAN, ${ }^{1}$ M. ZEBARJADI,${ }^{1}$ A. SHAKOURI, ${ }^{1}$ \\ J. KLEM ${ }^{2}$ G. PATRIZI, ${ }^{2}$ E.W. YOUNG ${ }^{2}$ and S.D. MUKHERJEE ${ }^{2}$ \\ 1.-Department of Electrical Engineering, University of California Santa Cruz, Santa Cruz, \\ CA 95064, USA. 2.-Sandia National Laboratories, Microsystems Science, Technology, \& \\ Components Center, Albuquerque, NM, USA. 3.—e-mail: wangxi@soe.ucsc.edu
}

The experimental characterization of single barrier heterostructure thermionic cooling devices at cryogenic temperatures is reported. The device studied was a cylindrical InGaAs microrefrigerator, in which the active layer was a $1 \mu \mathrm{m}$ thick $\mathrm{In}_{0.527} \mathrm{Al}_{0.218} \mathrm{Ga}_{0.255} \mathrm{As}$ heterostructure barrier with $n$-type doping concentration of $6.68 \times 10^{16} \mathrm{~cm}^{-3}$ and an $\mathrm{In}_{0.53} \mathrm{Ga}_{0.47} \mathrm{As}$ emitter/collector of $5 \times 10^{18} \mathrm{~cm}^{-3} n$-doping. A full field thermoreflectance imaging technique was used to measure the distribution of temperature change on the device's top surface when different current excitation values were applied. By reversing the current direction, we studied the device's behavior in both cooling and heating regimes. At an ambient temperature of $100 \mathrm{~K}$, a maximum cooling of $0.6 \mathrm{~K}$ was measured. This value was approximately one-third of the measured maximum cooling value at room temperature $(1.8 \mathrm{~K})$. The paper describes the device's structure and the first reported thermal imaging at cryogenic temperatures using the thermoreflectance technique.

Key words: Thermoelectric, thermionic, InAlGaAs barrier, cooling, thermoreflectance imaging

\section{INTRODUCTION}

Thermoelectric microrefrigerators are very attractive for die level thermal management and for cooling/temperature stabilization of individual devices in cryogenic sensors and imagers. They have significant volume and weight advantages when compared with conventional cooling solutions such as passive heat spreading and compressor based systems. Thermoelectric microrefrigerators are suitable for spot cooling, because they are small and are made of integrated circuit (IC) compatible materials. In addition, solid-state thermoelectric microrefrigerators are compact, quiet, and have no moving parts.

The utilization of a potential barrier in thermoelectric cooling devices gives an additional degree of

(Received July 31, 2008; accepted January 29, 2009;

published online February 21, 2009) freedom in comparison with the conventional Peltier modules, which are based on bulk properties. ${ }^{1}$ In a single potential barrier integrated device, when a forward electrical bias is applied, hot electrons are emitted from the cathode side, and reverse electron current is suppressed by a larger anode conduction band offset. Therefore, the device achieves a greater cooling efficiency. ${ }^{2-4}$

Typically, both bulk thermoelectric properties of the barrier material and evaporative thermionic emission from the cathode exist in a heterostructure integrated thermionic device. By varying the operating ambient temperature, one can alter the electron mean free path, to enhance ballistic transport, and thus observe the interplay between thermoelectric and thermionic contributions.

\section{SAMPLE DESCRIPTION}

The microrefrigerator device studied was a $20 \mu \mathrm{m}$ diameter, $1.3 \mu \mathrm{m}$ high, etched mesa of layer 


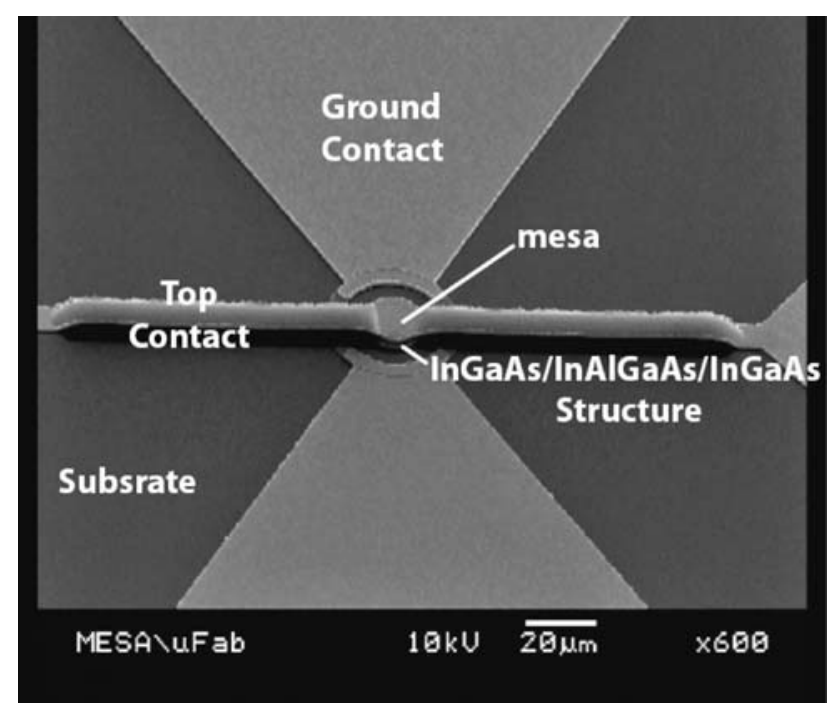

Fig. 1. SEM image of one example of an InGaAs/InAlGaAs microrefrigerator.

structure InGaAs/InAlGaAs/InGaAs, designed to be at flat band condition at $200 \mathrm{~K}$. The active layer was a $1 \mu \mathrm{m}$ thick $\operatorname{In}_{0.527} \mathrm{Al}_{0.218} \mathrm{Ga}_{0.255} \mathrm{As}$ heterostructure barrier, with an $n$-type doping concentration of $6.68 \times 10^{16} \mathrm{~cm}^{-3}$, and $0.3 \mu \mathrm{m}$ thick each $\mathrm{In}_{0.53} \mathrm{Ga}_{0.47} \mathrm{As}$ emitter/collector of $5 \times 10^{18} \mathrm{~cm}^{-3}$ $n$-doping. Several additional thin InGaAs/InAlGaAs superlattice layers were placed between the active layer and the $n+\operatorname{InP}$ substrate to provide a compositional grade to lower the electrical resistance of the InP/InGaAs transition. Figure 1 shows a scanning electron microscopy (SEM) image of a typical microrefrigerator.

\section{THERMOREFLECTANCE IMAGING METHOD}

The surface reflectivity of most materials has a weak dependence on the local surface temperature. ${ }^{5}$ Based on this dependence, the distribution of the temperature change on a material surface can be quantitatively described as:

$$
R=F(T, \lambda) T,
$$

where $R$ is the experimental measurement of the reflection coefficient of a surface at temperature $T$ and incident wavelength $\lambda$, and $F(T, \lambda)$ represents the function relating the measured value back to an absolute surface temperature. This function is nonlinear over a wide temperature range and depends on the material's parameters, such as phonon energies and band gap. It is possible to define the temperature dependency with a polynomial expansion and appropriate coefficients. However, for small changes, it is acceptable to limit the expansion to the linear term, since the change of reflectance per degree is typically within the range of $10^{-4}$ to $10^{-5}$ for semiconductors and an order of magnitude lower

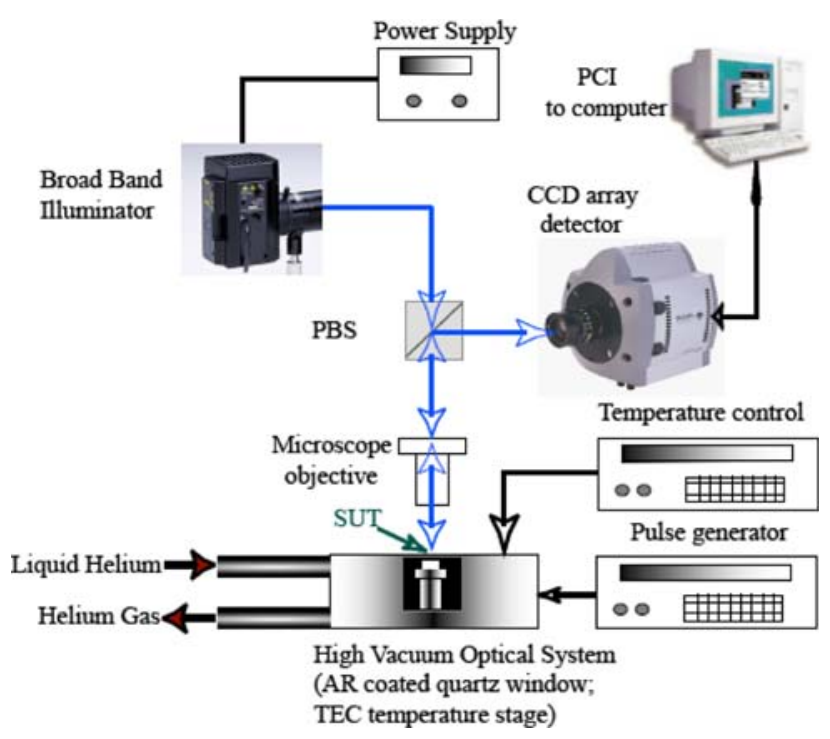

Fig. 2. Full-field thermoreflectance imaging setup. $\mathrm{PCl}$ : peripheral component interconnect; CCD: change-coupled device; PBS: polarized beam spliter; AR: anti-reflectance; TEC: thermal electric cooling.

for metals. We named the linear coefficient as the thermoreflectance coefficient, denoted by $C_{\mathrm{th}}$.

$$
C_{\text {th }}=\frac{1}{R_{0}} \frac{\partial R}{\partial T}
$$

$R_{1}$ and $R_{0}$ represent the excited $\left(T_{1}\right)$ and unexcited $\left(T_{0}\right)$ reflectivities. Solving for the temperature change of the surface, we obtain:

$$
\Delta T=\frac{\left(R_{1}-R_{0}\right)}{C_{\text {th }} \times R_{0}}
$$

Thermoreflectance is a very useful technique for non-contact, high spatial resolution, thermal imaging. The illumination wavelength can be chosen to allow a sub-micron spatial resolution, which is unavailable from diffraction-limited standard infrared (IR) imaging. ${ }^{6}$ Multiple-channel lock-in signal processing for each pixel allows fast full field detection without the lengthy time required by a scanning laser method. These advantages make thermoreflectance imaging suitable for cryogenic measurement, since the sample is placed inside a sealed chamber and the ambient temperature is kept stable during the imaging process.

Figure 2 illustrates the thermoreflectance imaging setup. Broad spectrum light emitted from an illuminator was focused onto the sample's top surface through a beam splitter and a high magnification objective. The reflected light that contained the information on the sample's surface temperature change was collected by a charge-coupled device (CCD) detector. The sample was kept in a high vacuum environment $\left(<1 \times 10^{-5}\right.$ Torr $)$ to prevent heat loss through air convection. A stable cryogenic temperature of $100 \mathrm{~K}$ was achieved by the combined use of liquid helium evaporation and a resistive 
heating stage inside the cryostat. During the entire measurement process, a thermometer was used to monitor the sample temperature. A fluctuation less than $1 \mathrm{~K}$ was observed.

\section{THERMOELECTRIC AND THERMIONIC COOLING}

The conventional thermoelectric effect is based on bulk properties of materials. When electrons flow from a material in which their average transport energy is less than the Fermi energy to another material in which their average transport energy is greater, they absorb thermal energy from the lattice, and this cools down the emitter side of the junction between the two materials. Reversal of the direction of current generates heat and creates a hot junction.

A great improvement of thermoelectric cooling efficiency is attributed to the heterostructure barrier, ${ }^{4-9}$ which takes advantage of the thermionic emission mechanism (selective emission of hot electrons over a barrier layer from cathode to anode). From a microscopic point of view, since the energy distribution of emitted electrons is almost exclusively above the Fermi energy, carrier-carrier and carrier-lattice scatterings tend to absorb the energy from the lattice in an effort to restore the quasi-equilibrium Fermi distribution in the cathode. Therefore, the emitter junction (cathode regime) is cooled. In addition, in the presence of thermionic emission and associated ballistic transport, Joule heating in the barrier is reduced, due to a reduction in scattering processes. Consequently, greater cooling efficiency is achieved.

\section{RESULTS AND DISCUSSION}

Figure 3 shows representative thermal images at a room temperature of $295 \mathrm{~K}$ and cryogenic temperature of $100 \mathrm{~K}$ in both cooling regime (current flows from the substrate to the device's top surface) and heating regime (current flows from the device's top surface to the substrate).

Figure 4 shows the temperature variations on the top surface of the microrefrigerator at room temperature in both cooling and heating regimes. At the ambient temperature of $295 \mathrm{~K}$, maximum cooling of $1.8 \mathrm{~K}$ was measured, while, at a cryogenic temperature of $100 \mathrm{~K}$, shown in Fig. 5, the maximum cooling was only $0.6 \mathrm{~K}$. Data analysis was carried out, based on the average temperature measured over the device's mesa area at each excitation current.

The sample was excited by a pulse current of frequency $f(f=106 \mathrm{~Hz})$. Within a cycle $\tau(\tau=1 / f)$, the electrical excitation can be represented by:

$$
I(t)= \begin{cases}I_{0} & 0 \leq|t|<\tau \\ 0 & \frac{\tau}{2}<|t| \leq \tau\end{cases}
$$

For a microrefrigerator, which is only a few microns in width and thickness, the time required to achieve thermal steady state is on the order of tens of microseconds. ${ }^{10}$ Therefore, it was anticipated that the device would achieve thermal steady state within each excitation cycle. The device's top surface temperature was determined by the balance between Peltier/thermionic cooling, Joule heating and the heat conduction within the device:

$$
\Delta T=\left(S T I+\chi R_{\mathrm{e}} I^{2}-Q\right) R_{\mathrm{th}},
$$

where $R_{\mathrm{e}}$ and $R_{\mathrm{th}}$ are electrical and thermal resistances of the device, respectively, and $\chi$ is the percentage of the Joule heating that flows back to the cold junction. $\chi$ is 0.5 for conventional thermoelectric cooling. $Q$ is the cooling power (amount of heat load being removed by the microrefrigerator). Under high vacuum and with low temperature surroundings, there is no thermal load on top of the device $(Q=0)$. If the geometry and material properties of the device are taken into consideration, as well as the biasing configuration, the device's top surface temperature can be approximated as:

$$
\begin{aligned}
\Delta T= & -R_{\text {th_sub }} S_{\mathrm{a}} T I-R_{\text {th_a }} S_{\mathrm{a}} T I+R_{\text {th_sub }} R_{\mathrm{e} \_\mathrm{a}} I^{2} \\
& +\chi R_{\text {th } \_\mathrm{a}} R_{\mathrm{e} \_\mathrm{a}} I^{2}+R_{\text {th_sub }} R_{\mathrm{e} \_ \text {sub }} I^{2},
\end{aligned}
$$

where $R_{\text {th_sub }}$ and $R_{\mathrm{e}_{\text {_sub }} \text { sub }}$ are the spreading thermal resistance and electrical resistance of the substrate, respectively. $R_{\mathrm{th} \_\mathrm{a}}$ and $R_{\mathrm{e} \_\mathrm{a}}$ are the thermal resistance and electrical resistance of the device's active region. $S_{\mathrm{a}}$ is the effective Seebeck coefficient of the active region. If we fit the cooling curves $(\Delta T$ versus I) as parabolas, the first and second order coefficients have physical meanings, as shown in Eq. 7.

$$
\begin{aligned}
\Delta T & =a I+b I^{2} \\
a & =-\left(R_{\text {th_sub }}+R_{\text {th_a }}\right) S_{\mathrm{a}} T \\
b & =\left(R_{\text {th_sub }}+\chi R_{\text {th_a }}\right) R_{\mathrm{e} \_\mathrm{a}_{\text {a }}}+R_{\text {th_sub }} R_{\text {e_sub }}
\end{aligned}
$$

The first order coefficient, $a$, is linearly related to the effective Seebeck coefficient of the active region. The second order coefficient, $b$, is linearly related to the percentage of the Joule heating that flows back to the cold junction. We can further derive the following expressions for $S_{\mathrm{a}}$ and $\chi$, as shown in Eqs. 8 and 9 .

$$
\begin{gathered}
S_{\mathrm{a}}=\frac{-a}{\left(R_{\text {th_sub }}+R_{\text {th_a }}\right) T} \\
\chi=\frac{b-R_{\text {th_sub }} R_{\text {e_a }}-R_{\text {th_sub }} R_{\mathrm{e} \_s u b}}{R_{\text {th } \_\mathrm{a}} R_{\mathrm{e} \_\mathrm{a}}}
\end{gathered}
$$

At lower temperature, the mean free path of electrons is increased. Since the total number of electrons emitted is fixed by the bias current and ambient temperatures, scattering events involved in thermoelectric cooling are diminished, leading to quasi-ballistic transport (more of thermionic cooling) through the barrier regime. This microscopic change also causes diminished Joule heating as 

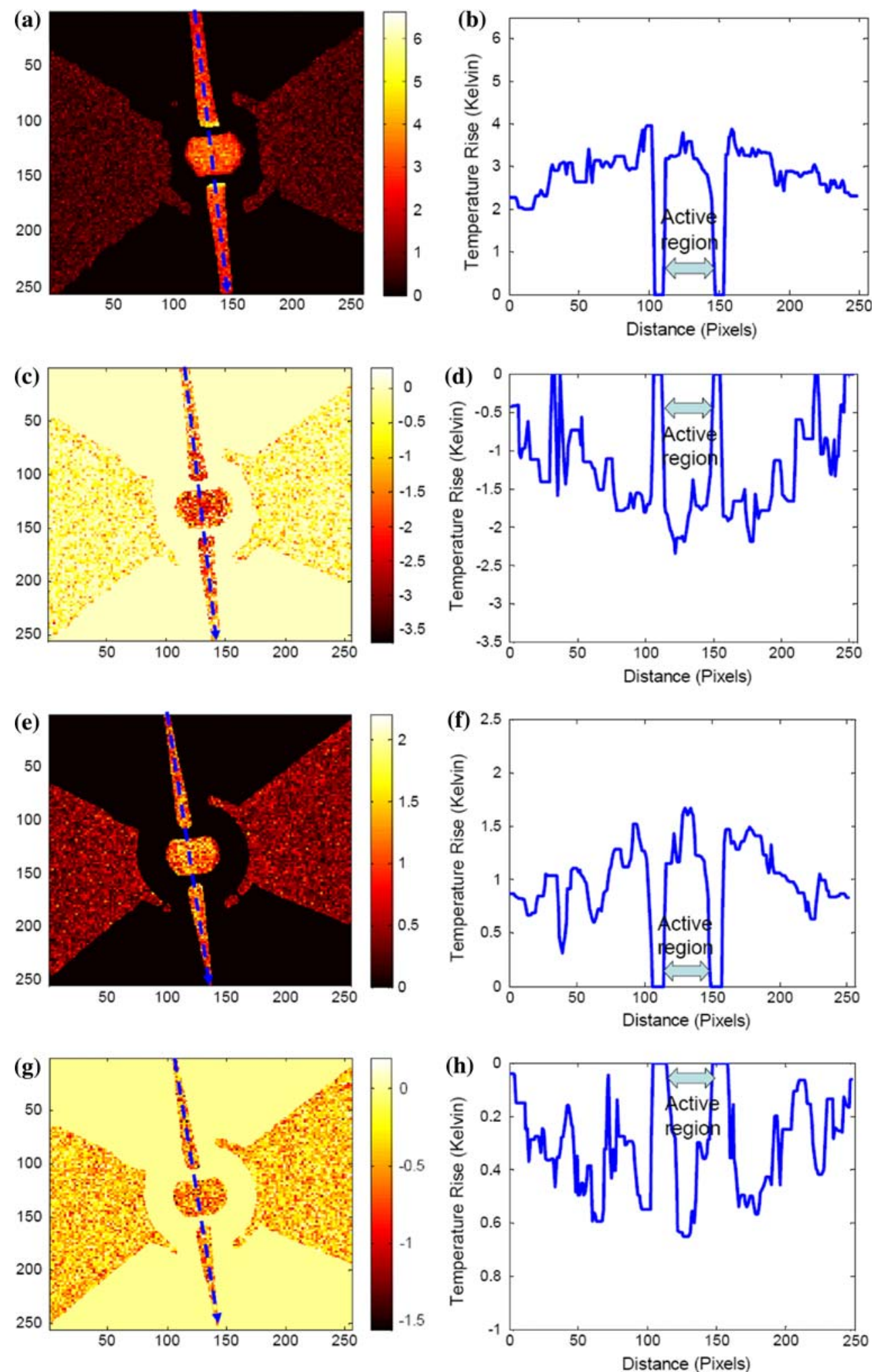

Fig. 3. Thermoreflectance images of the InGaAs microrefrigerator. $(\mathrm{a}, \mathrm{b})$ Thermal image and profile along the leads. $I=25 \mathrm{~mA}$ in the heating regime at $295 \mathrm{~K}$. (c, d) Thermal image and profile along the leads. $I=25 \mathrm{~mA}$ in the cooling regime (maximum cooling) at $295 \mathrm{~K}$. (e, f) Thermal image and profile along the leads. $I=15 \mathrm{~mA}$ in the heating regime at $100 \mathrm{~K} .(\mathrm{g}, \mathrm{h})$ Thermal image and profile along the leads. $I=15 \mathrm{~mA}$ in the cooling regime (maximum cooling) at $100 \mathrm{~K}$. 


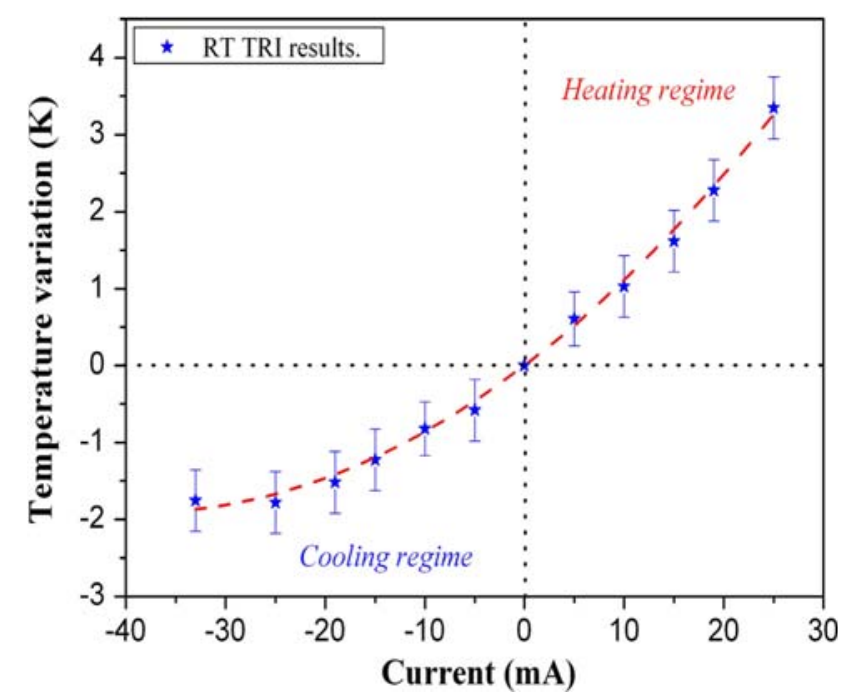

Fig. 4. Temperature variations on the top surface of the microrefrigerator at room temperature $(295 \mathrm{~K})$. RT: room temperature; TRI: thermal reflectance imaging.

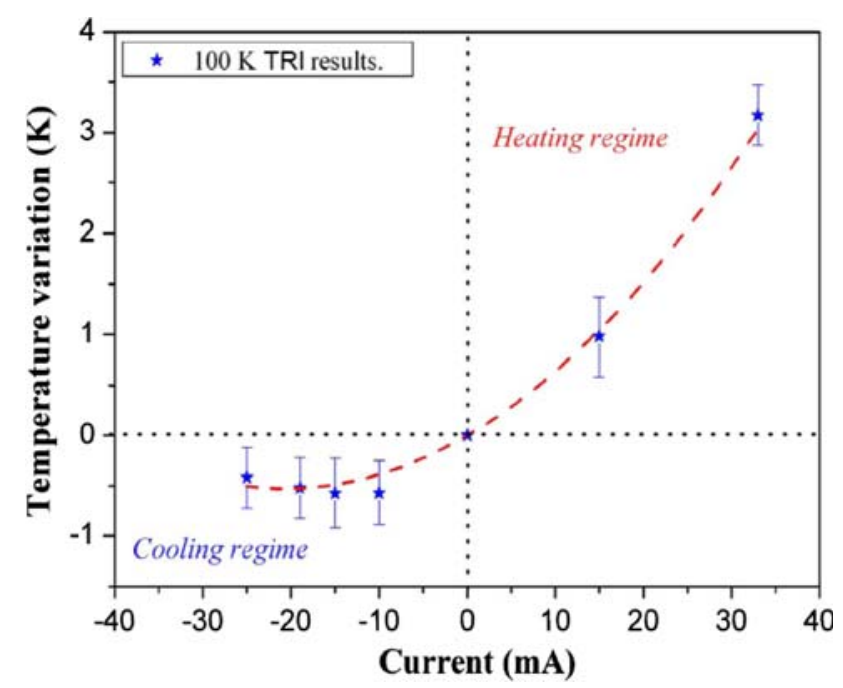

Fig. 5. Temperature variations on the top surface of the microrefrigerator at cryogenic temperature $(100 \mathrm{~K})$.

well as a shifted effective Joule heating center toward the anode. Therefore, the effective Seebeck coefficient is increased, and the percentage of the Joule heating that flows back to the cold junction is decreased. Both trends are indicated in the shape of the cooling curves. Parabolic fitting of the curves give the following expressions:

$$
\begin{aligned}
& \Delta T_{295 \mathrm{~K}}=0.09865 I+0.00128 I^{2} \\
& \Delta T_{100 \mathrm{~K}}=0.05108 I+0.00124 I^{2}
\end{aligned}
$$

One notes a significant change in the linear coefficient as a function of ambient temperature. This is expected, as the Peltier cooling is proportional to the absolute temperature. The change is less than the

\section{Heat/Current flow}

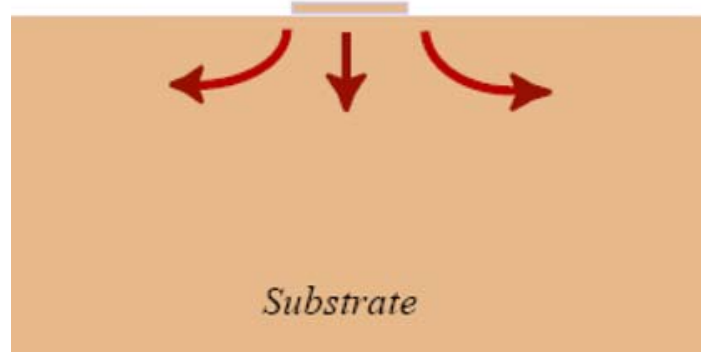

Fig. 6. Three-dimensional heat and current spreading in the substrate.

ratio of the two temperatures, which is due to the increase in the thermal conductivities and the change in the effective Seebeck coefficient at low temperatures. On the other hand, the quadratic coefficient is not changed significantly as a function of ambient temperature. Since the same thermal resistances appear in the expression of the quadratic coefficient (see Eq. 7), this effect is due to the change in electrical resistances and, possibly, a change in the fraction of the Joule heating transported back to the cold junction $(\chi)$. It is hard to extract all the material's parameters from these measurements, as, when the ambient temperature and material properties change, the 3D heat and current distribution in the substrate will change as well (see Fig. 6). More precise calculation would have to be done by using the finite element simulation and by analyzing different device sizes. These finite element simulation results, along with calculations of the temperature dependency of material properties, are to be presented in an upcoming paper.

\section{CONCLUSIONS}

The first thermal imaging at cryogenic temperatures using thermoreflectance technique is reported. Thermoelectric cooling and thermionic cooling in a single barrier InAlGaAs-based microrefrigerator were investigated experimentally. Maximum cooling of $1.8 \mathrm{~K}$ and $0.6 \mathrm{~K}$ over a $1 \mu \mathrm{m}$ thick barrier are reported at $295 \mathrm{~K}$ and $100 \mathrm{~K}$, respectively. These direct experimental results, along with further device modeling, will allow us to extract the material's properties and the contributions of thermoelectric cooling and thermionic cooling at different ambient temperatures.

\section{ACKNOWLEDGEMENTS}

The authors would like to thank Dr. James Christofferson of UC Santa Cruz, for the insightful discussions and his help in building the thermoreflectance imaging system, and Patrick Finnegan of L\&M Technologies (now at LMATA, Inc.), for fabricating the devices at Sandia National Laboratories. The authors would also like to acknowledge the partial support of the Interconnect Focus Center, one of five research centers funded under the Focus 
Center Research Program, a DARPA and Semiconductor Research Corporation program. Sandia is a multiprogram laboratory operated by Sandia Corporation, a Lockheed Martin Company, for the United States Department of Energy's National Nuclear Security Administration under contract DE-AC04-94AL85000.

\section{OPEN ACCESS}

This article is distributed under the terms of the Creative Commons Attribution Noncommercial License which permits any noncommercial use, distribution, and reproduction in any medium, provided the original author(s) and source are credited.

\section{REFERENCES}

1. M. Zebarjadi, A. Shakouri, and K. Esfarjani, Phys. Rev. B 74, 195331 (2006). doi:10.1103/PhysRevB.74.195331.

2. A. Shakouri, C. Labounty, J. Piprek, P. Abraham, and J.E. Bowers, Appl. Phys. Lett. 74, 88 (1999). doi:10.1063/ 1.122960 .
3. A. Shakouri and J.E. Bowers, Appl. Phys. Lett. 71, 1234 (1997). doi:10.1063/1.119861.

4. A. Shakouri, E.Y. Lee, D.L. Smith, V. Narayanamurti, and J.E. Bowers, Microscale Thermophys. Eng. 2, 37 (1998). doi:10.1080/108939598200097.

5. J. Christofferson and A. Shakouri, Rev. Sci. Instrum. 76, 024903-1 (2005).

6. J. Christofferson, K. Maize, Y. Ezzahri, J. Shabani, X. Wang, and A. Shakouri, J. Electron. Packag. 130, 041101 (2008).

7. X.F. Fan, G.H. Zeng, C. LaBounty, J.E. Bowers, E. Croke, C.C. Ahn, S. Huxtable, A. Majumdar, and A. Shakouri, Appl. Phys. Lett. 78, 1580 (2001). doi:10.1063/1.1356455.

8. C. LaBounty, A. Shakouri, P. Abraham, and J.E. Bowers, Opt. Eng. 39, 2847 (2000). doi:10.1117/1.1315567.

9. G.D. Mahan, J. Appl. Phys. 76, 4362 (1994). doi:10.1063/ 1.357324.

10. K. Maize, X. Wang, J. Park, J. Christofferson, S. Kang, and A. Shakouri, 1st International Symposium on Thermal Design and Thermophysics Property for Electronics, Tsukuba, Japan (18-20 June 2008). 